\title{
COMMENTS ON THE APPLICATIONS' OF PLANETARY NEBULAE RESEARCH
}

\author{
Manuel Peimbert \\ Instituto de Astronomía \\ Universidad Nacional Autonóma de México \\ Apartado Postal 70-264, México 04510 D.F., México
}

ABSTRACT. A discussion is given about some of the implications that advances in the study of PN are having in other areas of research such as: atomic physics, stellar formation rates, stellar evolution models, dust production, chemical evolution of galaxies, pregalactic helium abundance, stellar dynamics and the extragalactic distance scale.

\section{INTRODUCTION}

Professor Aller has given us a summary of the reviews and poster papers of this Symposium on the structure and evolution of planetary nebulae and their central stars. In my summary of the excellent presentations of this Symposium I Will concentrate on some of the results derived from PN that are relevant for other branches of astrophysics and of atomic physics. This is a biased summary since I will mainly cover recent results that are familiar to me.

\section{PN AS ATOMIC PHYSICS LABORATORIES}

Many of the review papers of the 1982 London Symposium on PN stress the relationship between atomic physics and PN (Flower 1983). In what follows I will mention some aspects of this relationship.

\subsection{Comparison of Ionization Structure Models with Observations}

A superb account of some of these aspects is presented in the review by Harrington (1988).

One of the most successful interactions between models and observations has occurred in the area of charge transfer reactions. Based on the comparison between ionization structure models and observations of NGC 7027 Pequignot et al. (1978, see also Pequignot 1980) concluded that the models were unable to account for several low and intermediate-excitation lines and proposed that charge transfer reactions with atomic hydrogen should be considered, in particular they suggested that the reaction

$$
\mathrm{O}^{+2}+\mathrm{H} \rightarrow \mathrm{O}^{+}+\mathrm{H}^{+}
$$

was fast, with $\beta\left(\mathrm{O}^{+2}\right) \simeq 8 \times 10^{-10} \mathrm{~cm}^{3} \mathrm{~s}^{-1}$. This suqaestion was confirmed by Butler et al. (1979) 577

S. Torres-Peimbert (ed.), Planetary Nebulae, 577-587.

(c) 1989 by the IAU. 
who found that $\beta\left(0^{+2}\right)=6 \times 10^{-10} \mathrm{~cm}^{3} \mathrm{~s}^{-1}$.

At the edge of extended objects of low degree of ionization, like NGC 6720 and NGC 7293 it has been found that the $\mathrm{Ne}^{++}$region coincides with the $\mathrm{O}^{+}$region (Hawley and Miller 1977, 1978; Hawley 1978); this result is explained by the large value of $\beta\left(O^{+2}\right)$ (Pequignot 1980).

\subsection{Electron Densities}

Since the pioneering paper by Osterbrock and Seaton (1957) on the electron densities in PN there has been an interplay between the observed line intensity ratios and the atomic parameters involved (e.g. Saraph and Seaton 1970).

Stanghellini and Kaler (1988) find that the densities derived from the [CI III] line intensities are higher by a factor of 2.6 relative to the densities derived from the [S II] and [O II] line intensities, which probably indicates that the $[\mathrm{Cl} I \mathrm{II}]$ atomic parameters should be revised.

\subsection{Abundances}

Different lines of the same element should yield the same ionic abundance. This has not been the case for $\mathrm{He}^{+}$abundances determined from different $\mathrm{He}$ l lines nor for $\mathrm{C}^{++}$abundances determined from the $\lambda C$ II recombination line and the $1909 \mathrm{C}$ III] collisionally excited line.

\subsection{1 $\mathrm{He}^{+}$Abundances}

The He I lines are affected by radiative transfer effects and collisions from the $2^{3} \mathrm{~S}$ metastable level. Cox and Daltabuit (1971) were the first to consider the effect of collisional excitation from the $2^{3} S$ level. Peimbert and Torres-Peimbert (1971) by comparing the abundances derived from the $\lambda \lambda 5876$ and 4471 lines concluded that the theoretical predictions had overestimated the collisional effects by about a factor of three. Berrington et al. (1985), see also Ferland (1986), based on an 11 state $a b$ initio computation obtained for $\lambda 5876$ results similar to those by Cox and Daltabuit. Peimbert and Torres-Peimbert (1987a) by comparing the abundances derived from the $\lambda \lambda 5876$ and $6678 \mathrm{He}$ I lines found again that the collisional cross sections had been overestimated by about factor of 3 or that the population of the $2^{3} S$ level was a factor of 3 smaller. Berrington and Kingston (1987), see also Clegg (1987), based on a 19 state ab initio computation found that the collisional effects were about a factor of 1.4 to 1.6 times smaller for $\lambda \lambda 5876$ and 6678 than those derived from the 11 state computation. Clegg (1987) estimated that collisional ionization reduced the population of the $2^{3} \mathrm{~S}$ level by a few percent, and Clegg and Harrington (1988) have estimated that under certain conditions photoionization can reduce the $2^{3} \mathrm{~S}$ level population by as much as $20 \%$. Peimbert and Torres-Peimbert (1987b) based on observations of $\lambda \lambda 5876,6678$ and 10830 still find that there is a difference of about 1.8 between the theoretical predictions and the observations; therefore an additional mechanism to depopulate the $2^{3} S$ level is needed, probably a charge exchange reaction that has not been considered. Higher quality observations of all the He I lines, in particular of $\lambda$ 10830 , are needed to advance in this problem.

\subsection{2 $\mathrm{C}^{++}$Abundances}

The $\mathrm{C}^{++}$abundance derived from $\lambda 4267$ is in general higher than that derived from $\lambda 1909$, the difference can reach in extreme cases an order of magnitude; nevertheless, it should be mentioned that there are objects that do not show this anomaly like IC 418, the Orion nebula and NGC 3918 (Torres-Peimbert et al. 1980; Clegg et al. 1987). General discussions of this problem have been given in the literature (e.g. Torres-Peimbert et al. 1980; Barker 1982; French 1983; Kaler 1986; Clegg 1988; see also references in these papers). Kaler (1986) has suggested that the discrepancy could be solved either by increasing the $C^{+}(\lambda$ 4267) effective recombination coefficient or by decreasing 
the target area of the $\mathrm{C}^{++}\left(2 \mathrm{~s}^{2}{ }^{1} \mathrm{~S}-2 \mathrm{~s} 2 \mathrm{p}^{3} \mathrm{P}\right)$ transition by about a factor of four. Alternatively, Peimbert (1983) has suggested that the discrepancy could by due to spatial variations in the electron temperature produced by inhomogeneities in the $\mathrm{C} / \mathrm{H}$ abundance ratio; the regions with higher $\mathrm{C} / \mathrm{H}$ abundance ratios would be at lower temperatures and would contribute preferentially to $\lambda 4267$ while the regions with lower $\mathrm{C} / \mathrm{H}$ abundance ratios would be at higher temperatures and would contribute preferentially to $\lambda(1909)$. Notice that the inhomogeneities could be due to carbon rich pockets embedded in a carbon poor medium, but could also be due to a $\mathrm{C} / \mathrm{O}$ ratio decreasing outwards.

There is observational evidence in favor of non homogeneous objects. PN are produced by several mass loss stages; the outer layers of the nebula are the least affected by stellar nucleosynthesis showing mainly products of hydrogen burning. Alternatively, continuous mass loss reveals layers of the central star where helium burning has occurred.

There is evidence that many of the AGB stars evolve from oxygen to carbon stars, for example J-type carbon stars, have silicate dust in their envelopes (see the review by Knapp 1988 and references therein), therefore for these objects we would expect an increase of the $\mathrm{C} / \mathrm{O}$ ratio from the outer layers inwards. Kudritzki and Méndez (1988) have discussed NGC 246 which has a peculiar PNN with a photosphere made mainly of $\mathrm{He}$ and $\mathrm{C}$ and with no trace of $\mathrm{H}$ (Husfeld 1986), but with normal nebular abundances (Heap 1975). Moreover, according to Méndez et al. (1986) $35 \%$ of all the spectroscopically well studied PNN belong to the extreme helium rich class.

Another non homogeneous object is NGC 40 where the central star is a Wolf-Rayet with $\mathrm{N}(\mathrm{He}) / \mathrm{N}(\mathrm{C}) \approx 15$ and no hydrogen (Benvenuti et al. 1982); the inner layers of the nebula show very strong C IV 1550 lines, which apparently imply an overabundance of carbon, and the outer layers of the nebula show almost normal $\mathrm{H}, \mathrm{C}, \mathrm{N}, \mathrm{O}$ abundances (Clegg et al. 1983). Moreover to explain the discrepancy between the temperature determined from the stellar nucleus and the much lower value inferred from the ionization of the nebula, Bianchi and Grewing (1987) have suggested the existence of a carbon curtain at the inner edge of the nebula.

Another extreme non homogeneous object is $\mathrm{A} 30$ which shows $\mathrm{He}$ and $\mathrm{C}$ rich condensations (Jacoby 1979; Hazard et al. 1980; Jacoby and Ford 1983; Peimbert 1983) with $N(H e) / N(H)=9 \pm 3$, $\mathrm{N}(\mathrm{He}) / \mathrm{N}(\mathrm{C})=13.2 \pm 4, \mathrm{~N}(\mathrm{He}) / \mathrm{N}(\mathrm{O})=132 \pm 42$ and $\mathrm{N}(\mathrm{He}) / \mathrm{N}(\mathrm{H})=86 \pm 29$.

Barker $(1982,1983,1984,1985,1986)$, has found that the $\lambda \lambda 4267,1909$ discrepancy becomes largest closer to the central stars of NGC 6720, NGC 7009, NGC 6853, NGC 3242 and NGC 7662; a $\mathrm{C} / \mathrm{O}$ ratio decreasing outwards in the parent star and carbon rich fast winds ejected in the later stages of evolution might also help to explain these results.

\subsection{Non-Linearity of the Detectors}

From observations of the 5007/4959 [O III], the 6583/6548 [N II], and the 4686/4541 He II line intensity ratios it is possible to check the linearity of a given detector; significant deviations from linearity have been found for IDS systems and for the ESA photon counting detector (e.g. Rosa 1985; Llebaria et al. 1986; Peimbert and Torres-Peimbert 1987a).

\section{FORMATION RATES AND THE INITIAL MASS FUNCTION}

To a first approximation the formation rates in the solar vicinity of: Miras, OH/IR stars, PN, white dwarfs and the main sequence turn off rate agree within a factor of 4! (Phillips 1988). This result is in agreement with the ideas about the genetic relation between Miras, OH/IR stars, PN and white dwarfs.Note that due to their larger scale height Phillips argues that not all the Miras become PN.

The PN formation rate depends on the fourth power of the distance scale adopted. Recent results by Barlow (1988), Kudritzky and Méndez (1988) and Mallik and Peimbert (1988) favor long distance scales, like that of Cudworth (1974), over short distance scales, like those of Cahn and Kaler (1971) and Daub (1982). 
The white dwarf and the PN formation rate derived from the Cudworth (1974) or the Mallik and Peimbert (1988) distance scales are in good agreement but are smaller than the formation rates derived from Miras or OH/IR stars. The determination of distances was discussed by Lutz (1988) and Terzian (1988) and to advance in this problem we need more distance determinations like those that could be derived from second epoch studies of the Lick survey or of radio data and those derived from spectroscopic studies of the central stars. I also consider that the accuracy in the estimation of the Mira and $\mathrm{OH} / \mathrm{IR}$ star formation rates should be improved.

\section{CONSTRAINTS ON STELLAR EVOLUTION MODELS}

Observations of PN provide strong constraints on the late stages of stellar evolution models.

The observed enrichment of $\mathrm{He}, \mathrm{C}$ and $\mathrm{N}$ in the PN envelopes of the solar vicinity, the halo, the SMC and the LMC have to be explained by stellar evolution models. Modest agreement between the observations and the predictions by Renzini and Voli (1981) has been obtained (e.g. Aller 1983; Kaler 1983; Peimbert 1985; Perinoto 1987; and references in these papers). The computations by Renzini and Voli have been made for two initial compositions and considering two parameters: $\alpha=$ $\mathrm{I} / \mathrm{H}$, the ratio of the mixing length to the pressure scale height, and $\eta$ which multiplied by the Reimers' rate (1975) gives the mass loss rate during the AGB phase. A new grid of models is needed for a larger range of $\alpha$ and $\eta$ values and of chemical compositions.

Dufour (1988) finds an inverse correlation between $\mathrm{O} / \mathrm{H}$ and stellar mass; the $\mathrm{O} / \mathrm{H}$ and $\mathrm{O} / \mathrm{N}$ ratios decrease in the sequence Type II PN $\rightarrow$ Type I PN $\rightarrow$ NGC 6164-5 $\rightarrow \eta$ Car. Apparently this is due to an increase in the $O$ to $N$ conversion with mass in the 1 to $40 M_{\odot}$ range.

The observed increase of the $\mathrm{C} / \mathrm{O}$ ratio in layers closer to the stellar center, see $\S 2.3 .2$, has to be explained by the models. In this respect, observations and predictions of $\mathrm{C}^{12} / \mathrm{C}^{13}$ ratios as a function of distance to the PN center would be very valuable (see the discussion by Knapp 1988).

NGC 6302 is $\mathrm{O}$ rich, it shows $\mathrm{OH}$ and $\mathrm{HCN}$ molecules (e.g. Rodríguez 1988), and it is probably one of the most massive PN known. Maybe this is a case of a hydrodinamical ejection of the whole envelope that would correspond to an object with $3 \leq M / M_{\odot} \leq 8$ (Renzini 1988).

PN envelopes are formed by stellar mass loss in the form of winds, just after the AGB phase a hot wind is emitted that compresses the material previously ejected by the ordinary wind (e.g. Kwok et al. 1978; Kwok 1982, 1983, 1988; Kahn 1983, 1988). The formation of toroids and bipolar structures around objects like NGC 2346, NGC 2440 and NGC 6302 has to be explained by the stellar evolution process. To explain these type of configurations different mass loss stages have been proposed from PNN in binary systems: (e.g. Morris 1981; Livio 1982; Renzini 1983; Iben and Tutukov 1988) and from individual PNN with high rotational velocities (Heap 1982; Calvet and Peimbert 1983).

Another important constraint on stellar evolution models is the observed fraction of PNN in binary systems. Bond (1988)and Méndez (1988) have discussed the estimates of the fraction of PNN in close and wide binaries respectively. Alternatively Iben and Tutukov (1988) have estimated the theoretical fraction of close binaries as well as the expected morphology and chemical composition of the envelopes.

The average central star mass derived from the sample by Kudritzky and Méndez (1988) is $0.69 \mathrm{M}_{\odot}$ and it is higher than those derived from the samples by Aller et al. (1987), $0.60 \mathrm{M}_{\odot}$, and Monk et al. (1988), $0.58 \mathrm{M}_{\odot}$. It is not clear why the difference is so large. Pottasch (1988), Tylenda (1988) and Barlow (1988) have also discussed the masses of PNN.

Sizes for the nebulae and magnitudes for the central stars of PN in the Magellanic Clouds from the Space Telescope will provide strong observational constraints to stellar evolution models (e.g. Barlow 1988). 


\section{DUST PRODUCTION}

It has been suggested that probably red giant stars are the main producers of interstellar dust in the Galaxy. Several authors have found that the $M_{\text {dust }} / M_{\text {gas }}$ ratio decreases by two to three orders of magnitude with the size of PN (Nata and Panagia 1981; Pottasch et al. 1984; Pottasch 1987; Lenzuni et al. 1987), result that contradicts the idea that PN are an important source of dust for the ISM. Lenzuni (1987) argues that the bulk of ISM dust grains originates from the winds of carbon stars and AGB stars before they may produce a PN.

Alternatively, Mallik and Peimbert (1988) from a distance scale independent sample of PN and considering the effect that the filling factor has in the gaseous mass determination find that $M_{\text {dust }} / M_{\text {gas }}$ does not vary substantially with the size of $P N$ and that the average $M_{\text {dust }} / M_{\text {gas }}$ value is equal to $6 \times 10^{-3}$. This value is similar to the value of $7 \times 10^{-3}$ estimated by Savage and Mathis (1979) for diffuse clouds in the Galaxy.

It is well known that $\mathrm{Fe}, \mathrm{Si}, \mathrm{Mg}$, and $\mathrm{Al}$ are depleted in gaseous envelopes of $\mathrm{PN}$ (e.g. Shields 1978, 1983; Pwa et al. 1986). Pwa et al. compute a mass fraction of $3.5 \times 10^{-3}$ for these elements if the abundances were solar. From the assumption that about $15 \%$ of the oxygen is locked in silicate cores and about $5 \% \pm 5 \%$ could be locked in polymer mantles (Meyer 1985) and if most of $\mathrm{Fe}, \mathrm{Si}$, $\mathrm{Mg}$ and $\mathrm{Al}$ were in dust grains we would expect a total $M_{\text {dust }} / \mathrm{M}_{\text {gas }}$ of about $5 \times 10^{-3}$ for solar abundances in excellent agreement with the value derived by Mallik and Peimbert (1988). Therefore if most of the mass ejected by intermediate mass stars becomes part of the PN then according to Mallik and Peimbert, it is still possible that most of the dust grains originate in PN.

\section{MODELS OF GALACTIC CHEMICAL EVOLUTION}

Based on the determination of the chemical composition of PN in: the solar vicinity, the halo of the Galaxy and other galaxies, it has been found that PN are enriching the ISM with $\mathrm{He}, \mathrm{C}$ and $\mathrm{N}$ (e.g. Kaler 1979; Peimbert and Serrano 1980; Torres-Peimbert et al. 1980; Aller and Czyzak 1983; Clegg 1988; Barlow 1988; and references in these articles). The observed values can be used as representative of the contribution of intermediate mass stars, those with $1 \leq M / M_{\odot} \leq 8$, to the enrichment of the ISM.

Tinsley (1978) considered that the solar $\mathrm{C} / \mathrm{O}$ ratio and estimates of nucleosynthesis in massive stars are consistent with a major source of $C$ arising from PN precursors. Sarmiento and Peimbert (1985), Mallik and Mallik (1985) and Matteucci (1986) found that most of the C in the ISM comes from intermediate mass stars.

Based on models of galactic chemical evolution an open discussion on the primary or secondary origin of $\mathrm{N}$ and on the relative importance of different stellar mass ranges in the $\mathrm{N}$ production is present in the literature (e.g. Edmunds and Pagel 1978; Peimbert and Serrano 1980; Serrano and Peimbert 1983; White and Audouze 1983; Matteucci 1986; Diaz and Tosi 1986; Forieri 1986; Pagel 1986, 1987a; Peimbert 1987). Peimbert (1987) finds that the $N$ enrichment produced by $P N$ of Types II and III is smaller in comparison to that produced by Type I PN, he also finds that the He enrichment produced by PN of Types II and III of the solar neighborhood is negligible in comparison to that produced by Type I PN.

The high $\Delta Y / \Delta Z$ values observed in the ISM are not easily explained from stellar evolution models and the observed IMF (see Peimbert 1986 and references therein). A very high relative contribution to the enrichment of the ISM by He-rich objects, like Type I PN and the Crab nebula (e.g. Davidson and Fesen 1985; Henry 1986; and references therein), is needed to be able to explain the observed $\Delta \mathrm{Y} / \Delta \mathrm{Z}$ values. 


\section{CHEMICAL COMPOSITION OF THE HALO}

The four known Type IV PN permit to discuss the chemical composition of the ISM at the time the stars in the halo were formed (see the discussion by Clegg 1988 and references therein).

Dinerstein (1987, see also Garnett and Dinerstein 1988) suggested that NGC 2242 might be a fifth halo PN. Torres-Peimbert et al. (1988) found that NGC 2242 and NGC 4361 have very similar properties: a) very high degree of ionization, b) very optically thin, they only trap about $4 \%$ of the Lyman continuum photons, and c) very similar surface brightnesses and electron densities. The abundances derived by Torres-Peimbert et al. are presented in Table 1.

TABLE 1. Total Abundances, $12+\log N(X) / N(H)$

\begin{tabular}{lllll}
\hline & NGC 2242 & NGC 4361 & Orion & Sun \\
\hline $\mathrm{He}$ & 10.99 & 11.00 & 11.01 & $\ldots$ \\
$\mathrm{O}$ & 8.05 & 7.94 & 8.65 & 8.92 \\
$\mathrm{Ne}$ & 7.39 & 7.25 & 7.80 & $\ldots$ \\
$\mathrm{Ar}$ & 6.19 & 6.07 & 6.65 & $\ldots$ \\
\hline
\end{tabular}

Other parameters for NGC 4361 are: distance from the plane $830 \mathrm{pc}$, mass of the central star $0.55 \mathrm{M}_{\odot}$, and ionized mass of the envelope, that corresponds to the total mass because the object is optically thin, $0.074 \mathrm{M}_{\odot}$; the distance from the plane for NGC 2242 is 870 pc (Kudritzky and Méndez 1988; Méndez et al. 1988; Torres-Peimbert et al. 1988). The two extremely low mass values for the central star and the envelope of NGC 4361 together with its distance from the plane, indicate that it is a population II object. Due to their location relative to the plane of the Galaxy NGC 2242 and NGC 4361 are not as extreme population II objects as the other four halo PN, on the other hand their $\mathrm{He} / \mathrm{H}, \mathrm{O} / \mathrm{H}$ and $\mathrm{Ne} / \mathrm{H}$ ratios are similar to those of DDDM-1 and $\mathrm{BB}-1$. From the values of Table 1 and the previous discussion, it follows that these PN should be classified as population II objects, either of Type III or of Type IV.

The central stars of NGC 4361 and NGC 1360 are very similar (Kudritzky and Méndez 1988; Méndez et al. 1988; Mc Carthy 1988). Therefore NGC 1360 might also be a Type IV PN; this object is located $510 \mathrm{pc}$ from the plane of the Galaxy and there are no abundance determinations for the gaseous envelope.

\section{GRADIENTS IN THE GALAXY AND M31}

PN present several advantages over $\dot{H} \|$ regions for the study of chemical abundance gradients across the disc of the Galaxy: a) we have a considerably larger sample available, b) since PN show a larger scatter in the direction perpendicular to the plane, it is possible to observe them in the optical domain over a larger range of galactocentric distances, c) in general small PN have higher surface brightnesses than galactic $H$ II regions observable in the optical domain; the disadvantages are: a) the initial $\mathrm{He}, \mathrm{C}, \mathrm{N}$, and $\mathrm{O}$ could have been modified by the evolution of their parent stars, b) they might show non circular orbits, and c) there is a spread in the ages of the stellar progenitors.

Maciel and Faúndez-Abans (1985) have derived a well defined electron temperature gradient from Type II galactic PN given by $\sim 600 \pm 120 \mathrm{~K} \mathrm{kpc}^{-1}$; this value is about 1.5 times larger than that derived from galactic $H$ II regions, the difference could be real since there are many different factors affecting each gradient. Faúndez-Abans and Maciel $(1986,1987 a)$ have derived abundance gradients for $\mathrm{He}, \mathrm{C}, \mathrm{N}, \mathrm{O}, \mathrm{Ne}, \mathrm{S}, \mathrm{Cl}$ and $\mathrm{Ar}$ relative to $\mathrm{H}$; their results are similar to those derived for $\mathrm{H}$ II regions and imply that most of the $\mathrm{PN}$ in their sample show orbits of relative low excentricity and that their age spread is small compared with the age of the disc of the Galaxy. 
By dividing Type II PN into two subtypes Type lla with $\log (\mathrm{N} / \mathrm{H})+12 \geq 8.0$ and Type Ilb with $\log (\mathrm{N} / \mathrm{H})+12<8.0$ Faúndez-Abans and Maciel (1987b) were able to obtain a much better correlation for the Type llb N/H gradient. This is probably due to two reasons: a) the smaller the $\mathrm{N} / \mathrm{H}$ value the smaller the $\mathrm{N}$ contamination due to stellar evolution, and $\mathrm{b}$ ) the $\mathrm{N}$ contamination might be due to two different processes, to the first dredge up that converts initial $\mathrm{C}$ into $\mathrm{N}$ and to a second source of $N$ which could be due to $O$ or to freshly made $C$, the second $N$ source might not be correlated with the initial $\mathrm{CNO}$ abundances but with the mass or with the binary nature of the nucleus of the PN.

Ford (1983) determined abundance gradients of $\mathrm{O} / \mathrm{H}$ and N/H for M31 that are considerably flatter than those for the Galaxy. Jacoby and Ford (1986) from the study of the chemical composition of three PN and an $\mathrm{H}$ II region in M31 found that M31 experienced considerably more enrichment of its interstellar medium during its early life than did the Galaxy, they also find that there is little evidence for a correlation between kinematics and metallicity in the halo of M31. More abundance determinations of PN in M31 should be carried out to advance in the discussion of the chemical evolution of M31.

\section{PREGALACTIC HELIUM ABUNDANCE}

A review on the relevance for cosmology and particle physics of the pregalactic or primordial helium abundance, $Y p$, has been given by Boesgaard and Steigman (1985). Clegg (1988) has discussed the derivation of $Y p$ and from the four halo $P N$ obtains that $Y p=0.23 \pm 0.03$. It should be mentioned that the observations of three of the four halo PN have not been corrected for the nonlinearity of the detector, the effect is very small, of the order of: $0: 01$ in $Y$, but it would go in the direction of increasing $Y p$.

It would be very important to determine the C abundance of NGC 2242 and NGC 4361 to estimate the amount of $\mathrm{He}$ due to stellar evolution and to be able to determine $\mathrm{Yp}$. It would also be very important to compute stellar evolution models for metal poor low mass stars to be able to predict the He enrichment due to stellar evolution.. Peimbert (1983) and Clegg (1988) based their He enrichment estimates on models and observations of objects with higher masses and higher heavy element content than those of Type IV PN.

Maciel $(1988 a, 1988 b)$ has estimated Yp by combining Type Ilb PN with galactic $\mathrm{H}$ II regions observed by Shaver et al. (1983) and with $\mathrm{O}$ poor extragalactic $\mathrm{H}$ II regions from the samples by Kunth and Sargent (1983), Pagel et al. (1986) and Pagel (1987b). He finds that $Y p=0.234 \pm 0.004$ $(1 \sigma)$ and that $\Delta Y / \Delta Z=3.5 \pm 0.3(1 \sigma)$. The $Y p$ value derive by Maciel depends mainly on the $O$ poor $\mathrm{H}$ II regions, while the $\Delta Y / \Delta Z$ ratio depends mainly on the $P N$. With respect to the $P N$ there are three corrections that should be made to the results by Maciel: a) some of the observations should be corrected for the non linearity of the detectors which will go in the direction of increasing $\Delta$ $Y / \Delta Z, b$ ) the collisional excitation from the $2^{3} S \mathrm{He}^{0}$ level (see $\S 2.3 .1$ ) should be taken into account, and c) even if the abundances of Type Ilb PN are not considerably affected by stellar evolution, all theoretical models predict at least some stellar He enrichment, this effect will go in the direction of decreasing $\Delta Y / \Delta Z$. These three effects are small and will affect the $\Delta Y / \Delta Z$ ratio by less than a factor of $\sim 1.3$.

\section{STELLAR DYNAMICS OF THE GALAXY AND OTHER GALAXIES}

PN have been used to study the stellar dynamics of the Galaxy and other galaxies. From the velocity and distance data for $252 \mathrm{PN}$ at galactocentric distances from 4 to $19 \mathrm{kpc}$, Schneider and Terzian (1983) derived the galactic rotation curve. They find a rising rotation curve with increasing galactocentric distances similar to those found in.most Sb and Sc galaxies (Rubin et al. 1980, 1982).

Masses, mass distributions and velocity dispersions have been obtained for galaxies as far away as a few megaparsecs. Barlow (1988) reviews the results for the SMC and Ford et al. (1988) 
for other extragalactic systems.

\section{THE EXTRAGALACTIC DISTANCE SCALE}

Ford et al. (1988) have given us an excellent account on the use of PN as standard candles. Objects used as standard candles should have approximately the same absolute flux, therefore we should analyze if the brightest PN in different galaxies are expected to have similar absolute fluxes. To a first approximation a PN emission line flux will depend on two quantities: on the number of Lyman continuum photons produced by the central star and on the ability of the nebula to trap these photons, i.e. if the nebula is optically thick or optically thin to Lyman continuum radiation.

The distance scales by Cudworth (1974) for optically thin and optically thick objects indicate that the brightest PN in the solar vicinity are optically thin which would make them poor standard candles because the absolute line fluxes would be a function not only of the central star properties but of the gas distribution in the nebula as well. Alternatively Gathier (1987) and Mallik and Peimbert (1988) have found that the brightest solar vicinity PN are optically thick and that the overwhelming majority of solar vicinity PN are optically thick contrary to the results by Cudworth and others. These results indicate that the emission line fluxes of the brightest PN depend directly on the properties of the central stars and not on the fraction of photons absorbed by the nebular shells.

Another property that makes good standard candles of PN is that the interstellar extinction can be easily determined from the Balmer line decrement.

It is a pleasure to acknowledge fruitful discussions with many of the participants to the Symposium, in particular with D.C.V. Mallik and S. Torres-Peimbert.

\section{REFERENCES}

Aller, L. H. 1983, in D. R. Flower (ed.), Planetary Nebulae, IAU Symp. No. 103, Dordrecht: Reidel, p.1. Aller, L. H. and Czyzak, S. J. 1983, Astrophys. J. Suppl. 51, 211.

Aller, L. H., Keyes, C. D., Maran, S. P., Gull, T. R., Michalitsianos, A G., and Stecher, T. P. 1987, Astrophys. J. 320, 159.

Barker, T. 1982, Astrophys. J. 253, 167.

Barker, T. 1983, Astrophys. J. 267, 630.

Barker, T. 1984, Astrophys. J. 284, 589.

Barker, T. 1985, Astrophys. J. 294, 193.

Barker, T. 1986, Astrophys. J. 308, 314.

Barlow, M. J. 1988, this volume.

Benvenuti, P., Perinotto, M., and Willis, A J. 1982, in C. de Loore and A J. Willis (eds.), Wolf-Rayet Stars, IAU Symp. No. 99, Dordrecht: Reidel, p. 453.

Berrington, K., Burke, P. G., Freitas, L. C. G., and Kingston, A. E. 1985, J. Phys. B 18, 4135.

Berrington, K. and Kingston, A. E. 1987, J. Phys. B 20, 6631.

Bianchi, L. and Grewing, M. 1987, Astron. Astrophys. 181, 85.

Boesgaard, A. and Steigman, G. 1985, Ann. Rev. Astron. Astrophys. 23, 319.

Bond, H. E. 1988, this volume.

Butler, S. E., Bender, C. F., and Dalgarno, A. 1979, Astrophys. J. 230, L59.

Cahn, J. H. and Kaler, J. B. 1971, Astrophys. J. Suppl. 22, 319.

Calvet, N. and Peimbert, M. 1983, Rev. Mexicana Astron. Astrofis. 5, 319.

Clegg, R. E. S. 1987, Mon. Not. R. astron. Soc. 229, 31 p.

Clegg, R. E. S. 1988, this volume.

Clegg, R. E. S. and Harrington, J. P. 1988, abstract of contributed paper, this volume.

Clegg, R. E. S., Harrington, J. P., Barlow, M.J., and Walsh, J. R. 1987, Astrophys. J. 314, 551.

Clegg, R. E. S., Seaton, M. J., Peimbert, M., and Torres-Peimbert, S. 1983, Mon. Not. R. astron. Soc. 
$205,417$.

Cox, D. P.and Daltabuit, E. 1971, Astrophys. J. 167, 257.

Cudworth, K.M. 1974, Astron. J. 79, 1384.

Daub, C. T. 1982, Astrophys. J. 260, 612.

Davidson, K. and Fesen, R. A. 1985, Ann. Rev. Astron. Astrophys. 23, 119.

Diaz,A. I. and Tosi, M. 1986, Astron. Astrophys. 158, 60.

Dinerstein, H. L 1987, comment made during the discussion of this Symposium.

Dufour,R. J. 1988, abstract of contributed paper, this volume.

Edmunds, M. G. and Pagel, B.E.J. 1978, Mon. Not. R. astron. Soc. 185, 77p.

Faúndez-Abans, M. and Maciel, W. J. 1986, Astron. Astrophys. 158, 228.

Faúndez-Abans, M. and Maciel, W. J. 1987a, Astrophys. Space Sci. 129, 353.

Faúndez-Abans, M. and Maciel, W. J. 1987b, Astron. Astrophys. 183, 324.

Ferland, G. J. 1986, Astrophys. J. 310, L67.

Flower, D.R., ed., 1983, Planetary Nebulae, IAU Symp. No. 103, Dordrecht: Reidel.

Ford, H.C. 1983, in D.R. Flower (ed.), Planetary Nebulae, IAU Symp. No. 103, Dordrecht: Reidel, p 443.

Ford, H.C., Ciardullo, R., Jacoby, G.H., and Hui,X. 1988, this volume.

Forieri, C. 1986, in C. Chiosi and A. Renzini (eds.), Spectral Evolution of Galaxies, Dordrecht: Reidel, p. 473.

French, H. B. 1983, Astrophys. J. 273, 214.

Garnett, D. R. and Dinerstein, H. L. 1988, Astron. J. 95, 119.

Gathier, R.1987, Astron. Astrophys. Suppl. 71, 245.

Harrington, J. P. 1988, this volume.

Hawley, S. A. 1978, Publ. Astron. Soc. Pacific 90, 370.

Hawley, S. A. and Miller, J. S. 1977, Astrophys. J. $212,94$.

Hawley, S. A. and Miller, J. S. 1978, Publ. Astron. Soc. Pacific 90, 39.

Hazard, C., Terlevich, R., Morton, D. C., Sargent, W. L. W., and Ferland, G. 1980, Nature 28 5, 463.

Heap, S. R. 1975, Astrophys. J. 196, 195.

Heap, S. R. 1982, in C. W. H. de Loore and A. J. Willis (eds.), Wolf-Rayet Stars, Observations, Physics and Evolution, Dordrecht: Reidel, p. 423.

Henry, R. B. C. 1986, Publ. Astron. Soc. Pacific 98, 1044.

Husfeld, D. 1986, Ph. D. thesis, Universität München.

Iben, I., Jr. and Tutukov, A. V. 1988, this volume.

Jacoby, G. H.1979, Publ. Astron. Soc. Pacific 91, 754.

Jacoby, G. H. and Ford, H. C. 1983, Astrophys. J. 266, 298.

Jacoby, G. H. and Ford, H. C. 1986, Astrophys. J. 304, 490.

Kahn, F. 1983, in D. R. Flower (ed.), Planetary Nebulae, IAU Symp. No. 103, Dordrecht: Reidel, p. 305.

Kahn, F. 1988, this volume.

Kaler, J. B. 1979, Astrophys. J. 228, 163.

Kaler, J. B. 1983 , in D. R. Flower (ed.), Planetary Nebulae, IAU Symp. No. 103, Dordrecht: Reidel, p. 245.

Kaler, J. B. 1986, Astrophys. J. 308, 337.

Knapp, G. 1988, this volume.

Kudritzki, R. P. and Méndez, R. H. 1988, this volume.

Kunth, D. and Sargent, W. L. W. 1983, Astrophys. J. 273, 81.

Kwok, S. 1982, Astrophys. J. 258, 280.

Kwok, S. 1983, in D. R. Flower (ed.), Planetary Nebulae, IAU Symp. No. 103, Dordrecht: Reidel, p. 293.

Kwok, S. 1988, this volume.

Kwok, S., Purton, C. R., and Fitzgerald, P. M. 1978, Astrophys. J. $219, \mathrm{~L} 125$. 
Lenzuni, P. 1987, in A. Preite-Martinez (ed.), Planetary and Protoplanetary Nebulae: from IRAS to ISO, Dordrecht: Reidel, p. 254.

Lenzuni, P., Natta, A. and Panagia, N. 1987, in A. Preite-Martinez (ed.), Planetary and Proto-planetary Nebulae: from IRAS to ISO, Dordrecht: Reidel, p. 249.

Livio, M. 1982, Astron. Astrophys. 105, 37.

Llebaria, A., Nieto, J. L., and di Serego Alighieri, S. 1986, Astron. Astrophys. 168, 389.

Lutz, J. 1988, this volume.

Maciel, W. J. 1988a, abstract of contributed paper, this volume.

Maciel, W. J. 1988b, Astron. Astrophys., in press.

Maciel, W. J. and Faúndez-Abans, M. 1985, Astron. Astrophys. 149, 365.

Mallik, D. C. V. and Mallik, S. V. 1985, J. Astrophys. Astron. 6, 113.

Mallik, D. C. V. and Peimbert, M. 1988, Rev. Mexicana Astron. Astrofis., submitted.

Matteucci, F. 1986, Publ. Astron. Soc. Pacific 98, 973.

Mc Carthy, J. K. 1988, private communication.

Méndez, R. H. 1988, this volume.

Méndez, R. H., Kudritzki, R. P., Herrero, A., Husfeld, D., and Groth, H. G. 1988, Astron. Astrophys. 190, 113.

Méndez, R. H., Miguel, C. H., Heber, U., and Kudritzki, R. P. 1986, in K. Hunger, D. Schönberner, and N. Kameswara (eds.), Hydrogen Deficient Stars and Related Objects, IAU Coll. 87, Dordrecht: Reidel p. 323.

Meyer, J. P. 1985, Astrophys. J. Suppl. 57, 151.

Monk, D. J., Barlow, M. J., and Clegg, R. E. S. 1988, abstract of contributed paper, this volume.

Morris, M. 1981, Astrophys. J. 249, 572.

Natta, A and Panagia, N. 1981, Astrophys. J. 248, 189.

Pagel, B. E. J. 1986, in E. Vangioni-Flan et al. (eds.), Advances in Nuclear Astrophysics, France: èditions Frontiéres, p. 53.

Pagel, B. E. J. 1987a, in G. Gilmore and B. Carswell (eds.), The Galaxy, Dordrecht: Reidel, p. 341.

Pagel, B. E. J. 1987b, unpublished.

Pagel, B. E. J., Terlevich, R. J., and Melnick, J. 1986, Publ. Astron. Soc. Pacific 98, 1005.

Peimbert, M. 1983, in P. Shaver and D. Kunth (eds.), Primordial Helium, Garching: ESO, p. 267.

Peimbert, M. 1985, Rev. Mexicana Astron. Astrofis. 10, 125.

Peimbert, M. 1986, Publ. Astron. Soc. Pacific 98, 1057.

Peimbert, M. 1987, in A. Preite-Martinez (ed.), Planetary and Protoplanetary Nebulae: from IRAS to ISO, Dordrecht: Reidel, p. 91.

Peimbert, M. and Serrano, A. 1980, Rev. Mexicana Astron. Astrofis. 5, 9.

Peimbert, M. and Torres-Peimbert, S. 1971, Astrophys. J. 168, 413.

Peimbert, M. and Torres-Peimbert, S. 1987a, Rev. Mexicana Astron. Astrofis. 14, 540.

Peimbert, M. and Torres-Peimbert, S. 1987b, Rev. Mexicana Astron. Astrofis. 15, 117.

Pequignot, D. 1980, Astron. Astrophys. $81,356$.

Pequignot, D., Aldrovandi, S. M. V., and Stasińska, G. 1978, Astron. Astrophys. 63, 313.

Perinotto, M. 1987, in A Preite-Martinez (ed.), Planetary and Protoplanetary Nebulae: from IRAS to ISO, Dordrecht: Reidel, p. 13.

Phillips, P. 1988, this volume.

Pottasch, S. R. 1987,in S. Kwok and S. R. Pottasch.(eds.), Late Stages of Stellar Evolution, Dordrecht: Reidel, p. 355 .

Pottasch, S. R. 1988, this volume.

Pottasch, S. R. et al. 1984, Astron. Astrophys. 138, 10.

Pwa, R., Pottasch, S. R., and Mo, J. E. 1986, Astron. Astrophys. 164, 184.

Reimers, D. 1975, Mem. Soc. Roy. Sci. Liege, 6e Ser. 8, 369.

Renzini, A. 1983, in D. R. Flower (ed.), Planetary Nebulae, IAU Symp. No. 103, Dordrecht: Reidel, p. 267. 
Renzini, A. 1988, this volume.

Renzini, A. and Voli, M. 1981, Astron. Astrophys. 94, 175.

Rodríguez, L.F. 1988, this volume.

Rosa, M. 1985, ESO Messenger 39, 15.

Rubin, V. C., Ford, W. K, and Thonnard, N. 1980, Astrophys. J. 238, 471.

Rubin, V. C., Ford, W. K., Thonnard, N., and Burstein, D. 1982, Astrophys. J. 26 1, 439.

Saraph, H.E. and Seaton, M.J. 1970, Mon. Not. R. astron. Soc. 148, 367.

Sarmiento, A. and Peimbert, M. 1985, Rev. Mexicana Astron. Astrofis. 11, 73.

Savage, B. D. and Mathis, J. S. 1979, Ann. Rev. Astron. Astrophys. 17, 73.

Schneider, S. E. and Terzian, Y. 1983, Astrophys. J. 274, L61.

Seaton, M. J. and Osterbrock, D. E. 1957, Astrophys. J. 125, 66.

Serrano, A. and Peimbert, M. 1983, Rev. Mexicana Astron. Astrofis. 8, 117.

Shaver, P. A., Mc Gee, R. X., Newton, L. M., Danks, A. C., and Pottasch, S. R. 1983, Mon. Not. R. astron. Soc. 204, 53.

Shields, G. A. 1978, Astrophys. J. 219, 565.

Shields, G. A. 1983, in D. R. Flower (ed.), Planetary Nebulae, IAU Symp. No. 103, Dordrecht: Reidel, p. 259.

Stanghellini, L and Kaler, J. B. 1988, abstract of contributed paper, this volume.

Terzian, Y. 1988, this volume.

Tinsley, B.M. 1978, in Y. Terzian (ed.), Planetary Nebulae, Observations and Theory, IAU Symp. No. 76, Dordrecht: Reidel, p. 341.

Torres-Peimbert, S., Peimbert, M., and Daltabuit, E. 1980, Astrophys. J. 238, 133.

Torres-Peimbert, S., Peimbert, M., and Peña, M. 1988, Astron. J., submitted.

Tylenda, R. 1988, this volume.

White, S. D. and Audouze, J. 1983, Mon. Not. R. astron. Soc. 203, 603. 